Lepr Rev (1998) 69, 106-109

\title{
Editorial
}

\section{WHY MULTIDRUG THERAPY FOR MULTIBACILLARY LEPROSY CAN BE SHORTENED TO 12 MONTHS}

To overcome the serious threat posed by the widespread emergence of dapsone resistance, ${ }^{1}$ and to increase the therapeutic effect in chemotherapy of leprosy, a World Health Organization (WHO) Study Group in 1981 recommended multidrug therapy (MDT) for the treatment of leprosy. $^{2}$ It was recommended that, for the purpose of treating different categories of patients with various bacterial loads, leprosy be classified as paucibacillary (PB) and multibacillary (MB), and that two drugs, monthly rifampicin (RMP) and daily dapsone (DDS), be prescribed for the treatment of PB leprosy, and three drugs-daily DDS and clofazimine (CLO) together with monthly RMP plus a supplemental higher dose of CLOfor MB leprosy. The duration of MDT for PB leprosy is 6 months; whereas for MB leprosy, it was recommended that MDT should be given for at least 2 years and preferably be continued up to skin-smear negativity. ${ }^{2}$ Because of the promising results of 24-month treatment, the WHO Study Group recommended, at its second meeting in 1994, that all MB leprosy should be treated for 24 months. ${ }^{3}$ The MDT regimens have proved to be highly effective and well tolerated by the patients. ${ }^{4,5}$ At the beginning of 1997 , more than 8.4 million leprosy patients had been cured by MDT. ${ }^{5}$

However, from the operational point of view, the duration of MDT is still too long, especially for MB leprosy. The long duration of treatment has become one of the major obstacles in implementing MDT, particularly in areas where the health infrastructure is poor or the accessibility is difficult. It would facilitate the implementation of MDT among all patients who need treatment if the duration of MDT could be further shortened without significantly compromising its efficacy.

To avoid relapse caused by spontaneously occurring RMP-resistant mutants and to minimize the relapse due to drug-susceptible organisms after stopping MDT, the appropriate duration of MDT for MB leprosy is the time required to reduce the size of viable bacterial population to such an extent that RMP-resistant mutants are completely eliminated and the great majority of drug-susceptible organisms are killed. To date, due to technical constraints, we are unable to determine directly, with any laboratory tool, whether or not the RMP-resistant mutants are still present in the hosts, or whether the drug-susceptible organisms are reduced to a negligible level. However, the following information may be useful to define the appropriate duration of MDT for MB leprosy.

First of all, the definition of MB leprosy has become much broader since 1981, when the Study Group designed the MDT regimen. Originally, MB leprosy referred to those patients 
who had a bacterial index (BI) of $\geq 2$ at any site in the initial skin smears. ${ }^{2}$ A few years later, the WHO Expert Committee on Leprosy at its 6th Meeting modified the definition that all skin smear positive cases should be classified as MB leprosy; ${ }^{4}$ and the Second WHO Study Group further recommended that, when the classification is in doubt, the patients should be treated as having MB leprosy. ${ }^{3}$ Then, because of the lack of dependable skin-smear facilities in most leprosy programmes, the WHO Expert Committee on Leprosy at its 7th Meeting proposed that patients could be classified on clinical grounds only, and that MB leprosy should refer to those having more than five skin lesions. ${ }^{5}$ These modifications have resulted in the classification of many cases that would otherwise be PB leprosy as MB leprosy, and the proportion of MB leprosy among newly detected cases has increased from $20.8 \%$ in 1985 to $30.9 \%$ in $1996 .{ }^{6}$ A more important finding is that, unlike in the early 1980 s when all newly detected $\mathrm{MB}$ cases were skin smear positive, the proportion of smear positive cases among newly detected MB leprosy cases in 1996 was less than half. Among 142,844 newly detected MB cases from the 16 major leprosy endemic countries, it was estimated that 69,449 $(48.6 \%)$ were skin smear positive, and only $24,216(17 \cdot 0 \%)$, or one-sixth, of MB cases have a $\mathrm{BI}$ of $>3 .^{7}$ Because the bacterial loads of the majority of MB patients currently classified are significantly smaller than those in the past, the overall requirements of chemotherapy for $\mathrm{MB}$ leprosy may also be less.

Secondly, the results from both routine control programmes ${ }^{8}$ and from research projects ${ }^{9}$ have demonstrated that the relapse rates after MDT were very low, about $0 \cdot 2 \%$ annually, among MB leprosy cases. Similar results have been obtained after 2-year fixed duration MDT. ${ }^{10-14}$ The low relapse rates indicate that there is enough room for further shortening the duration of MDT to less than 24 months. Although some reports suggested that relapse rates after MDT could be significantly higher among MB patients with a high initial BI, i.e. the average $\mathrm{BI} \geq 4 \cdot 0,{ }^{15,16}$ because such patients have become relatively scarce in the field, ${ }^{7}$ the total number of relapses by them contributed to a leprosy control programme will be small. The programmes should accept the few relapses that may occur from patients with a high initial BI and treat those patients who do relapse with a further course of MDT.

Thirdly, the major role of the DDS-CLO component of the MDT regimen for MB leprosy is to ensure the elimination of the spontaneously occurring RMP-resistant mutants, estimated to be no greater than $10^{4}$ organisms in an untreated patient with lepromatous leprosy, ${ }^{17}$ before stopping chemotherapy. The results from both nude mouse experiments ${ }^{18}$ and a clinical trial $^{19}$ have demonstrated that the bactericidal effect of the DDS-CLO component was significantly greater than expected; 3 months of daily treatment with DDS-CLO component alone killed more than $99.999 \%$ of viable Mycobacterium leprae, ${ }^{18}$ suggesting that all the spontaneously occurring RMP-resistant mutants are likely to be eliminated by 3-6 months of treatment with the DDS-CLO component in the MDT regimen.

Fourthly, in a multicentre, double-blind trial organized by the Steering Committee on Chemotherapy of Mycobacterial Diseases (THEMYC) of the UNDP/World Bank/WHO Special Programme for Research and Training in Tropical Diseases, MB patients with initial $\mathrm{BI} \geq 2$ were randomized into four groups of about 500 patients each, and two of the four groups were treated, respectively, with 24-month or 12-month MDT. After 4-6 years of follow-up from intake, or 3-5 years after stopping treatment with the 12-month regimen, not a single relapse has been detected among the two groups, which suggests that the 12-month MDT is as effective as the standard 24-month MDT regimen (THEMYC Steering Committee, unpublished data). The efficacy of various durations of MDT has also been compared in a clinical trial in Malawi, in which $305 \mathrm{MB}$ cases were randomly allocated into two groups 
and treated, respectively, with 18 or 30 months of MDT. ${ }^{20}$ After stopping treatment, the mean duration of follow-up was 3 years, with a maximum of 6 years. In both groups, the BI continued to fall, and fell to 0 by 60 months of follow-up. No relapse was observed in either group and the percentage of patients who developed new disabilities was similar. It was concluded that 18-month MDT may be sufficient for the treatment of MB leprosy.

Finally, information on the clinical and bacteriological progress of defaulted MB cases may shed some light on the efficacy of MDT with duration shorter than the standard one. In one study, ${ }^{21} 41$ defaulted MB cases were retrieved. They had been treated with MDT for a mean duration of 7 months (range 3-13 months), and had not taken treatment after defaulting. By the time the patients were retrieved, from less than 1 year to more than 5 years after drop-out, all 41 patients showed clinical improvement, and 29 (71\%) became smear negative, while the BI was stationary in five $(12.2 \%)$ cases. In another series of patients, ${ }^{7}$ who were skin smear positive before defaulting, 139 and 95 of them had been treated, respectively, with $<12$ months and 13-23 months of MDT before defaulting. By the time the patients were retrieved, after a mean duration of drop-out for 7.6 and 7.5 years, respectively, only $11(7.9 \%)$ patients from the former and six $(6.3 \%)$ patients from the latter group were still smear positive. Not only were the positive rates very similar between the two groups, but neither differed significantly from those $(3.3 \%)$ of 761 patients who had completed 24 months of MDT and were examined 4 years later. Although one has to be cautious in interpreting the information from the retrospective analyses, because the records are often incomplete, the sample size is relatively small and the pretreatment characteristics of the patients between the groups may not be comparable, they do suggest that treatment with less than 12 months of MDT exhibited promising therapeutic effects among the majority of MB patients.

On the basis of all the available information, the WHO Expert Committee on Leprosy concluded, at its latest meeting of 1997, that it is possible that the duration of the MDT regimen for MB leprosy could be further shortened to 12 months. ${ }^{5}$ This conclusion has been well-accepted by almost all the leprosy control programmes of the major endemic countries and is being implemented. Of course, during the transitional period from 24-month MDT to 12-month, a series of operational issues should be addressed, such as providing guidelines for the transition, revising national manuals, introducing a new reporting system, and improving the detection and treatment of leprosy reactions after completion of treatment. However, compared with the earlier days when MDT was introduced, in most countries now the leprosy control programme managers and their field staffs are more experienced, and they are able to handle these operational issues without too much difficulty.

Faculté de Médecine Pitié-Salpétriére

B. $\mathrm{J}_{\mathrm{I}}$

91 Boulevard de l'Hôpital

75634 Paris Cedex 13

France

\section{References}

1 Ji B. Drug resistance in leprosy-a review. Lepr Rev, 1985; 56: 265-278.

2 WHO Study Group. Chemotherapy of leprosy for control programmes. WHO Technical Report Series, No. 675. World Health Organization, Geneva, 1982.

${ }^{3}$ WHO Study Group. Chemotherapy of leprosy. WHO Technical Report Series No. 847. World Health Organization, Geneva, 1994. 
${ }^{4}$ WHO Expert Committee on Leprosy. Sixth Report. WHO Technical Report Series, No. 768. World Health Organization, Geneva, 1988.

5 WHO Expert Committee on Leprosy. Seventh Report. WHO Technical Report Series, No. 874. World Health Organization, Geneva, 1998 (in press).

6 World Health Organization. Global case-detection trend in leprosy. Weekly Epid Rec, 1997; 72: 173-180.

${ }^{7}$ World Health Organization. Shortening duration of treatment of multibacillary leprosy. Weekly Epid Rec, 1997; 72: $125-132$

8 WHO Leprosy Unit. Risk of relapse in leprosy. WHO document (WHO/CTD/LEP/94.1), 1994.

${ }^{9}$ Beck-Bleumink M. Relapses among leprosy patients treated with multidrug therapy, experience in the leprosy control program of the All Africa Leprosy and Rehabilitation Training Centre (ALERT) in Ethiopia; practical difficulties with diagnosing relapses; operational procedures and criteria for diagnosing relapses. Int J Lepr, 1992; 60: $421-435$.

10 Vijayakumaran P, Jesudasan K, Manimozhi N. Fixed-duration therapy (FDT) in multibacillary leprosy: efficacy and complications. Int J Lepr, 1996; 64: 123-127.

11 Jesudasan K, Vijayakumaran P, Manimozhi N, Jeyarajan T, Rao PSS. Absence of relapse within 4 years among 34 multibacillary patients with high BIs treated for 2 years with MDT. Int J Lepr, 1996; 64: 133-135.

12 Li H, Hu L, Wu P, Luo J, Liu X. Fixed-duration multidrug therapy in multibacillary leprosy. Int J Lepr, 1997; 65: $230-237$.

13 Li H, Hu L, Huang W, Liu G, Yuan I, Jin Z, Li X, Li J, Yang Z. Risk of relapse in leprosy after fixed-duration multidrug therapy. Int J Lepr, 1997; 65: 238-245.

14 Dasananjali K, Schreuder PAM, Pirayavaraporn C. A study on the effectiveness and safety of the WHO/MDT regimen in the Northeast of Thailand; a prospective study, 1984-1996. Int J Lepr, 1997; 65: 28-36.

15 Jamet P, Ji B, and Marchoux Chemotherapy Group. Relapse after long-term follow-up of multibacillary patients treated by WHO multidrug regimen. Int J Lepr, 1995; 63: 195-201.

16 Girdhar BK. Personal communication, 1996.

$17 \mathrm{Ji} \mathrm{B}$, Grosset JH. Recent advances in the chemotherapy of leprosy (Editorial). Lepr Rev, 1990; 61: 313-329.

$18 \mathrm{Ji} \mathrm{B}$, Perani EG, Petinom C, Grosset JH. Bactericidal activities of combinations of new drugs against Mycobacterium leprae in nude mice. Antimicrob Agents Chemother, 1996; 40: 393-399.

19 Ji B, Jamet P, Perani EG, Sow S, Liemhardt C, Petinom C, Grosset JH. Bactericidal activity of single dose of clarithromycin plus minocycline, with or without ofloxacin, against Mycobacterium leprae in patients. Antimicrob Agents Chemother, 1996; 40: 2137-2141.

20 Ponnighaus JM, Boerrigter G. Are 18 doses of WHO/MDT suf ficient for multibacillary leprosy? Results of a trial in Malawi. Int J Lepr, 1995; 63: 1-7.

21 Ganapati R, Shroff HJ, Gandewar KL, Rao BRP, Pai RR, Kute AS, Fernandes TX, Revankar CR, Pawar PL. Five year follow-up of multibacillary leprosy patients after fixed duration chemotherapy. Quaderni di cooperazione sanitaria, 1992; 12: 223-229. 